\title{
Laplacian ideals, arrangements, and resolutions
}

\author{
Anton Dochtermann • Raman Sanyal
}

Received: 27 February 2013 / Accepted: 1 February 2014 / Published online: 28 March 2014

(C) Springer Science+Business Media New York 2014

\begin{abstract}
The Laplacian matrix of a graph $G$ describes the combinatorial dynamics of the Abelian Sandpile Model and the more general Riemann-Roch theory of $G$. The lattice ideal associated to the lattice generated by the columns of the Laplacian provides an algebraic perspective on this recently (re)emerging field. This binomial ideal $I_{G}$ has a distinguished monomial initial ideal $M_{G}$, characterized by the property that the standard monomials are in bijection with the $G$-parking functions of the graph $G$. The ideal $M_{G}$ was also considered by Postnikov and Shapiro (Trans Am Math Soc 356:3109-3142, 2004) in the context of monotone monomial ideals. We study resolutions of $M_{G}$ and show that a minimal-free cellular resolution is supported on the bounded subcomplex of a section of the graphical arrangement of $G$. This generalizes constructions from Postnikov and Shapiro (for the case of the complete graph) and connects to work of Manjunath and Sturmfels, and of Perkinson et al. on the commutative algebra of Sandpiles. As a corollary, we verify a conjecture of Perkinson et al. regarding the Betti numbers of $M_{G}$ and in the process provide a combinatorial characterization in terms of acyclic orientations.
\end{abstract}

Keywords Graph Laplacian · Chip-firing · Lattice ideal · Initial ideal · G-parking function · Cellular resolution · Graphical arrangement · Acyclic orientation

Mathematics Subject Classification (2010) $\quad 05 E 40 \cdot 05 C 25 \cdot 13 \mathrm{D} 02 \cdot 52 \mathrm{C} 35$

\footnotetext{
A. Dochtermann $(\varangle)$

Department of Mathematics, University of Miami, Coral Gables, FL, USA

e-mail: anton.dochtermann@gmail.com; anton@math.miami.edu

R. Sanyal

Fachbereich Mathematik und Informatik, Freie Universität Berlin, Berlin, Germany

e-mail: sanyal@math.fu-berlin.de
} 


\section{Introduction}

Let $G=(V, E)$ be an undirected and connected graph with vertex set $V=[n+1]=$ $\{1,2, \ldots, n+1\}$. The Laplacian $\mathcal{L}(G)$ of $G$ is the symmetric $(n+1) \times(n+1)$ matrix that encodes the dynamics of the chip-firing game on the graph $G$. More recently the Laplacian has been central to the study of a discrete version of the Riemann-Roch theorem for graphs, where chip-firing serves as the graph-theoretic notion of linear equivalence of divisors.

In this paper we are interested in a certain class of ideals arising from $\mathcal{L}(G)$. We fix a field $\mathbb{K}$ and consider the lattice ideal $I_{G} \subset \mathbb{K}\left[x_{1}, \ldots x_{n+1}\right]$ associated to $\mathcal{L}(G)$. By definition $I_{G}$ is generated by binomials of the form $\mathbf{x}^{\mathbf{u}}-\mathbf{x}^{\mathbf{v}}$ where $\mathbf{u}, \mathbf{v} \in \mathbb{N}^{n+1}$, and $\mathbf{u}-\mathbf{v}$ are in the lattice spanned by the rows of $\mathcal{L}(G)$. Following the lead of [12] we call this ideal the toppling ideal of the graph $G$; it was first introduced by Perkinson et al. in [16].

After fixing the vertex $n+1$, the ideal $I_{G}$ has a distinguished monomial initial ideal $M_{G}$ with the property that the standard monomials of $M_{G}$ are in bijection with the so-called G-parking functions. This monomial ideal was first studied by Postnikov and Shapiro [17] in the context of monotone monomial ideals and their deformations and can be defined by an explicit combinatorial rule (see below). As is illustrated in [12], the ideal $M_{G}$ has interesting connections to the Riemann-Roch theory of $G$.

In each of the papers $[12,16,17]$ various free resolutions of the ideals $I_{G}$ and $M_{G}$ are considered. In the case that $G=K_{n+1}$ is a complete graph on $n+1$ vertices, it is shown in [17] that the monomial ideal $M_{G}$ has a minimal cellular resolution supported on the first barycentric subdivision of a $(n-1)$-simplex. This fact is used in [17], where the authors describe resolutions of the lattice ideal $I_{G}$ in the case that $G$ is a saturated graph. Indeed in this case the monomial ideal in question is generic, and the resolution coincides with the Scarf complex of $M_{G}$. By results of [15], this resolution lifts to the Scarf complex of the lattice ideal $I_{G}$. In [17] the authors show that the barycentric subdivision of an $(n-1)$-dimensional simplex supports a resolution of $M_{G}$ for an arbitrary graph $G$ on $n$ vertices. However, these resolutions are typically far from minimal and thus directly accessible homological information (such as (graded) Betti numbers) is limited. In both [17] and [12] the issue of finding a minimal resolution for the case of a general graph $G$ is left as an open question.

In this paper we describe a simple and explicit minimal cellular resolution of the monomial ideal $M_{G}$. The polyhedral complex supporting the resolution is obtained from the graphical hyperplane arrangement $\mathcal{A}_{G}$ associated to $G$. More precisely, the intersection of $\mathcal{A}_{G}$ with a certain affine subspace yields the essential affine hyperplane arrangement $\tilde{\mathcal{A}}_{G}$. Our main result (Theorem 3) is that $\mathcal{B}_{G}$, the bounded subcomplex of $\tilde{\mathcal{A}}_{G}$, supports a minimal-free resolution of the monomial ideal $M_{G}$. As a corollary of our result, we verify a conjecture of Perkinson et al. regarding the Betti numbers of $M_{G}$. In particular the Betti numbers of $M_{G}$ enumerate acyclic orientations of certain contractions of the graph $G$ (see Corollary 3).

In their work on Laplacian lattice ideals, Manjunath and Sturmfels [12] demonstrate how the duality involved in the discrete Riemann-Roch for certain graphs can be expressed in terms of $M_{G}^{*}$, the ideal Alexander dual to $M_{G}$ with respect to the canonical 
monomial of $G$. Our constructions also lead to an explicit description of a (co)cellular resolution of the ideal $M_{G}^{*}$ for all graphs $G$; see Sect. 6.1.

This collaboration began in Berlin in the summer of 2012, and the results of this paper were first presented in the Combinatorics seminar at the University of Miami in September 2012. While this paper was being prepared, the two preprints [11] and [14] were posted on the arXiv announcing similar results. Both papers employ purely algebraic/combinatorial methods while our perspective is that of geometric combinatorics. In recent conversations with the authors of [14] we were made aware of their independent work-in-progress involving cellular resolutions.

\section{Graphs, $G$-parking functions, and monomial ideals}

Throughout the paper we let $G=(V, E)$ be a finite, undirected graph on the vertex set $V=[n+1]=\{1,2, \ldots, n+1\}$ and edge set $E$. We assume that $G$ is connected and without loops but with possibly parallel edges, i.e., multiple edges between vertices $i$ and $j$.

We let $\mathcal{L}(G)$ denote the Laplacian of $G$. Recall that $\mathcal{L}(G)$ is the symmetric $(n+$ $1) \times(n+1)$ matrix with $\mathcal{L}(G)_{i j}=-\mid$ \{edges between $i$ and $\left.j\right\} \mid$ if $i \neq j$ and equal to the degree of $i=j$, otherwise. We will denote by $\Lambda(G)$ the sublattice of $\mathbb{Z}^{n}$ generated by the rows of $\mathcal{L}(G)$. The Laplacian has been studied in various combinatorial settings including spectral graph theory [7]. Since $G$ is assumed to be connected, $\mathcal{L}(G)$ has a one-dimensional kernel spanned by the vector $(1,1, \ldots, 1)^{t}$. The celebrated MatrixTree theorem (see [7, Sect. 13.2]) asserts that $|\operatorname{det} \tilde{\mathcal{L}}(G)|$ is the number of spanning trees of the graph $G$. This is an application of the Binet-Cauchy theorem to the truncated Laplace matrix $\tilde{\mathcal{L}}(G)$, the matrix obtained by deleting the $(n+1)$ st (or any other) row and column from $\mathcal{L}(G)$. More recently, the Laplacian of $G$ has appeared in the context of a discrete Riemann-Roch theory for graphs [1], where it encodes the dynamics of the so-called chip-firing moves (the discrete analog of linear equivalence of divisors).

We fix a field $\mathbb{K}$ and let $\mathbb{K}\left[x_{1}, x_{2}, \ldots, x_{n+1}\right]$ denote the polynomial ring in $n+1$ generators. Associated to our graph $G$ we let $I_{G}$ denote the lattice ideal associated to $\Lambda(G)$. By definition $I_{G}$ is generated by binomials of the form $\mathbf{x}^{\mathbf{u}}-\mathbf{x}^{\mathbf{v}}$, where $\mathbf{u}-\mathbf{v}$ is in the lattice $\Lambda(G)$, generated by the columns of $\mathcal{L}(G)$,

$$
I_{G}=\left\langle\mathbf{x}^{\mathbf{u}}-\mathbf{x}^{\mathbf{v}}: \mathbf{u}, \mathbf{v} \in \mathbb{N}^{n+1}, \mathbf{u}-\mathbf{v} \in \Lambda(G)\right\rangle
$$

Here we use the notation $\mathbf{x}^{\mathbf{u}}=x_{1}^{u_{1}} x_{2}^{u_{2}} \cdots x_{n+1}^{u_{n+1}}$. The ideal $I_{G}$ is called the toppling ideal of the graph $G$ in [12] and [16].

The binomial ideal $I_{G}$ has a distinguished monomial initial ideal $M_{G}$, characterized by the property that the standard monomials of $M_{G}$ are given by the $G$-parking functions. The ideals $M_{G}$ have also been studied in the context of monotone monomial ideals in [17] and can be described explicitly as follows. For any nonempty subset $I \subseteq[n]$ we define the monomial

$$
m_{I}:=\prod\left\{x_{i}: i j \in E, i \in I, j \in[n+1] \backslash I\right\}=\prod_{i \in I} x_{i}^{d_{I}(i)},
$$


where $d_{I}(i)$ denotes the number of edges from the vertex $i$ to a vertex in $[n+1] \backslash I$. Now define $M_{G}$ to be the ideal in $R=\mathbb{K}\left[x_{1}, \ldots, x_{n}\right]$ generated by all $m_{I}$, as $I$ ranges over all nonempty subsets of $[n]$ :

$$
M_{G}=\left\langle m_{I}: \emptyset \neq I \subseteq[n]\right\rangle \subseteq \mathbb{K}\left[x_{1}, \ldots, x_{n}\right] .
$$

Since $G$ is connected and $n+1 \notin I, M_{G}$ is a proper monomial ideal in $R$. For a subset $I \subseteq[n+1]$, let us denote by $G[I]$, the vertex-induced subgraph, i.e., the graph with vertex set $I$ and edges $\{i j \in E: i, j \in I\}$.

Proposition 1 The monomial $m_{I}$ is a minimal generator of $M_{G}$ if and only if $G[I]$ and $G\left[I^{c}\right]$ are connected.

Proof Suppose $G[I]=G\left[I_{1}\right] \uplus G\left[I_{2}\right]$. Then $m_{I}=m_{I_{1}} \cdot m_{I_{2}}$. Similarly, if $G\left[I^{c}\right]=$ $G\left[J_{1}\right] \uplus G\left[J_{2}\right]$ and $n+1 \in J_{2}$, then $m_{I \cup J_{1}}$ divides $m_{I}$. Thus, $G[I]$ and $G\left[I^{c}\right]$ are connected for every minimal generator.

Conversely, assume that $I \subseteq[n]$ satisfies the condition and assume that $m_{J}$ divides $m_{I}$ with $m_{J} \neq m_{I}$. Observe that $J$ cannot be a subset of $I$. By the definition of $m_{I}$ given in (2) above we see that

$$
J \subseteq I \Longrightarrow d_{J}(j) \geq d_{I}(j) \text { for all } j \in J
$$

Thus, let $j \in J \backslash I$. Along a path from $j \in I^{c}$ to $n+1$ inside $G\left[I^{c}\right]$, there is an edge $s t \in E$ such that $s \in J$ but $t \in J^{c}$. Since $s \in I^{c}$, we have $d_{I}(s)=0$. But then $d_{J}(s)>0=d_{I}(s)$ which contradicts that $d_{J}(i) \leq d_{I}(i)$ for all $i \in[n]$.

According to [4], a G-parking function is a function $\phi:[n] \rightarrow \mathbb{Z}_{\geq 0}$ such that for every $\emptyset \neq I \subseteq[n]$, there is an $i \in I$ such that $0 \leq \phi(i)<d_{I}(i)$. It is easily seen that $\phi:[n] \rightarrow \mathbb{Z}_{\geq 0}$ is a $G$-parking function if and only if $\mathbf{x}^{\phi}=x_{1}^{\phi(1)} x_{2}^{\phi(2)} \cdots x_{n}^{\phi(n)} \notin M_{G}$. Thus, the $G$-parking functions of $G$ are exactly the nonvanishing monomials in $\mathbb{K}[\mathbf{x}] / M_{G}$. It is known [6] that the number of $G$-parking functions (and hence the $\mathbb{K}$-vector space dimension of $\mathbb{K}[\mathbf{x}] / M_{G}$ ) equals the number of spanning trees of $G$ which, as we have seen, is given by $|\operatorname{det} \tilde{\mathcal{L}}(G)|$.

To realize $M_{G}$ as an initial ideal of $I_{G}$, we first fix a spanning tree $T$ of $G$ rooted at the vertex $n+1$ and choose an ordering of the variables that is a linear extension of $T$. More concretely, we choose a total order $\preceq$ of $\left\{x_{1}, \ldots, x_{n+1}\right\}$ that satisfies $x_{i} \succ x_{j}$ if in $T$ the vertex $i$ lies on the unique path from $n+1$ to $j$. We then take the reverse lexicographic term order for this ordering of variables. We call any such monomial ordering a spanning tree order, borrowing the term from [12].

Example 1 If $G=K_{n+1}$ is the complete graph on $n+1$ vertices, we can take the usual reverse lexicographic term order. The ideal $M_{G}$ is the tree ideal on $n$ variables, as described in [13, Sect. 4.3.4]. In this case the standard monomials of $M_{G}$ are given by the classical parking functions studied in combinatorics, i.e., sequences of nonnegative integers $\left(a_{1}, a_{2}, \ldots, a_{n}\right)$ with the property that, when placed in weakly ascending order, sit below a staircase: $a_{i} \leq\left|\left\{j: a_{j} \leq a_{i}\right\}\right|$ for all $i \in[n]$. The maximal parking functions are given by the permutations of $n$, which also correspond to the generators of the ideal that is Alexander dual to $M_{G}$. 
Example 2 A graph $G$ is said to be saturated if $G$ has $u_{i j}>0$ edges between any two vertices $i \neq j$. In [12] it is shown that if $G$ is a saturated graph on $n+1$ vertices then the ideal $I_{G}$ is a generic lattice ideal. For such graphs an explicit set of $2^{n}-1$ binomial generators is given that form a Gröbner basis of $I_{G}$ with respect to reverse lexicographic order.

We next introduce the example that will be used throughout the paper.

Example 3 (Running example) Let $G$ be the 4 -cycle with vertices $\{1,2,3,4\}$ and edges $\{12,23,34,14\}$. The Laplacian $\mathcal{L}(G)$ is the $4 \times 4$ matrix given below.

$$
\mathcal{L}(G)=\left[\begin{array}{rrrr}
2 & -1 & 0 & -1 \\
-1 & 2 & -1 & 0 \\
0 & -1 & 2 & -1 \\
-1 & 0 & -1 & 2
\end{array}\right]
$$

The ideal $I_{G}$ is given by

$$
I_{G}=\left\langle x_{1}^{2}-x_{2} x_{4}, x_{2}^{2}-x_{1} x_{3}, x_{3}^{2}-x_{2} x_{4}, x_{4}^{2}-x_{1} x_{3}, x_{1} x_{2}-x_{3} x_{4}, x_{2} x_{3}-x_{1} x_{4}\right\rangle .
$$

The monomial ideal $M_{G}$ is given by

$$
M_{G}=\left\langle x_{1}^{2}, x_{2}^{2}, x_{3}^{2}, x_{1} x_{2}, x_{1}^{2} x_{3}^{2}, x_{2} x_{3}, x_{1} x_{3}\right\rangle .
$$

The generator $m_{\{1,3\}}=x_{1}^{2} x_{3}^{2}$ is redundant, so $M_{G}=\left\langle x_{1}^{2}, x_{2}^{2}, x_{3}^{2}, x_{1} x_{2}, x_{2} x_{3}, x_{1} x_{3}\right\rangle=$ $\left\langle x_{1}, x_{2}, x_{3}\right\rangle^{2}$.

\section{Cellular resolutions}

Let $R=\mathbb{K}\left[x_{1}, \ldots, x_{n}\right]$ denote the polynomial ring on $n$ variables with the standard $\mathbb{Z}^{n}$-grading given by $\operatorname{deg}\left(\mathbf{x}^{a}\right)=a \in \mathbb{Z}_{\geq 0}^{n}$ for any monomial $\mathbf{x}^{a}$. For any graded $R$-module $M$, a $\mathbb{Z}^{n}$-graded free resolution of $M$ is an exact sequence

$$
0 \leftarrow M \stackrel{\phi_{1}}{\longleftarrow} F_{1} \stackrel{\phi_{2}}{\longleftarrow} \ldots \stackrel{\phi_{r}}{\longleftarrow} F_{r} \leftarrow 0
$$

where each $F_{i}$ is a graded free $R$-module

$$
F_{i} \cong \bigoplus_{\sigma \in \mathbb{Z}^{n}} R(-\sigma)^{\beta_{i, \sigma}}
$$

and where each $\phi_{i}$ is a graded map. The resolution is called minimal if each of the $\beta_{i, \sigma}$ is minimal among all graded free resolutions of $M$. In this case the $\beta_{i, \sigma}=\beta_{i, \sigma}(M)$ are called the finely or $\mathbb{Z}^{n}$-graded Betti numbers of the module $M$. The $\mathbb{Z}$-graded Betti numbers of $M$ are given by

$$
\beta_{i, j}(M)=\sum_{|\sigma|=j} \beta_{i, \sigma}(M)
$$


Fig. 1 The (minimal) free resolution of $M_{G}$ for $G=K_{4}$ is supported on $\mathcal{B}\left(\Delta_{2}\right)$. Vertices are labeled by the subsets $I \subseteq[n]$ corresponding to generators $m_{I}$

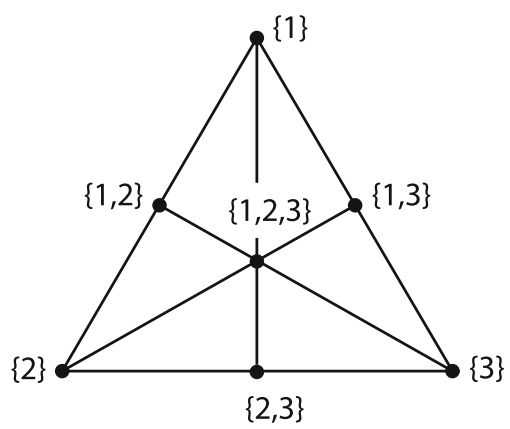

where $|\sigma|=\sigma_{1}+\sigma_{2}+\cdots+\sigma_{n}$. And finally, for each integer $i$, the $i$ th (ungraded) Betti number of $M$ is given by

$$
\beta_{i}(M)=\sum_{j} \beta_{i, j}(M) .
$$

A labeled polyhedral complex is a polyhedral complex $\mathcal{X}$ together with an assignment $a_{F} \in \mathbb{N}^{n}$ to each face $F \in \mathcal{X}$ such that

$$
\left(a_{F}\right)_{i}=\max \left\{\left(a_{G}\right)_{i}: G \subset F\right\} .
$$

for all $i=1,2, \ldots, n$. Let $\mathcal{X}$ be a labeled polyhedral complex and let

$$
M=M_{\mathcal{X}}=\left\langle\mathbf{x}^{a_{F}}: F \in \mathcal{X}\right\rangle \subseteq \mathbb{K}\left[x_{1}, \ldots, x_{n}\right]
$$

be the monomial ideal generated by the labels. Clearly, $M_{\mathcal{X}}$ is generated by the 0-cells of $\mathcal{X}$. For $\sigma \in \mathbb{N}^{n}$, let $\mathcal{X}_{\leq \sigma} \subseteq \mathcal{X}$ be the subcomplex of faces $F$ for which $a_{F} \leq \sigma$ componentwise. Bayer and Sturmfels [3] described how labeled complexes can encode resolutions of $M$, that is, when the chain complex $\mathcal{F}_{\mathcal{X}}$ of $\mathcal{X}$ gives rise to a $\mathbb{Z}^{n}$-graded resolution-a cellular resolution—of $M$. We refer to [13] for further details from which the following criterion is taken.

Proposition 2 ([13, Proposition 4.5]) Let $\mathcal{X}$ be a labeled polyhedral complex and let $M=M_{\mathcal{X}} \subset \mathbb{K}\left[x_{1}, \ldots, x_{n}\right]$ be the associated monomial ideal. Then $\mathcal{X}$ supports $a$ cellular resolution $\mathcal{F}_{\mathcal{X}}$ of $M$ if and only if the subcomplex $\mathcal{X}_{\leq \sigma}$ is $\mathbb{K}$-acyclic for all $\sigma \in \mathbb{N}^{n}$. The resolution is minimal if $a_{F} \neq a_{G}$ for all faces $F \subset G \in \mathcal{X}$.

Resolutions of $I_{G}$ and $M_{G}$ provide a new perspective on the duality expressed in the Riemann-Roch theory of the graph $G$ via the notion of Alexander duality. The Betti numbers of $M_{G}$ have combinatorial interpretations in terms of the graph $G$, and, as we will see, also relate to certain well-studied geometric complexes.

In [12] the authors consider resolutions of $I_{G}$ and $M_{G}$. They show that in the case of saturated graphs $G$, the toppling ideal $I_{G}$ has a minimal cellular resolution supported on what we will denote as $\mathcal{B}\left(\Delta_{n-1}\right)$, the first barycentric subdivision of an $(n-1)$-dimensional simplex (Fig. 1). 
In this case the complex $\mathcal{B}\left(\Delta_{n-1}\right)$ can be lifted to the Scarf complex associated to the (in this case generic) lattice ideal $I_{G}$. This extends a result from [17], where it is shown that the monomial ideal $M_{G}$ for $G=K_{n+1}$ has a resolution supported on the same complex $\mathcal{B}\left(\Delta_{n-1}\right)$.

For an arbitrary (not necessarily saturated) graph $G$, it is shown in [12] that the complex $\mathcal{B}\left(\Delta_{n-1}\right)$ supports a generally nonminimal cellular resolution of $I_{G}$. This leads to a formula for the Betti numbers of $M_{G}$ in terms of the ranks of the reduced homology of certain induced subcomplexes of $\mathcal{B}\left(\Delta_{n-1}\right)$, although it is not clear how one might obtain an explicit expression.

The description of a minimal resolution of $M_{G}$ for an arbitrary graph $G$ is stated as an open question in both [12] and [17]. In [16] the authors provide a conjecture for the Betti numbers of $M_{G}$ in the context of combinatorial data arising from Riemann-Roch theory of $G$. In Sect. 6 we confirm this conjecture.

\section{Chip-firing and superstable configurations}

The Laplacian $\mathcal{L}(G)$ describes the dynamics of the so-called chip-firing game or Abelian Sandpile Model for $G$. We outline the basic construction here and refer to [16] for further details. In this context, let us choose $n+1$ as a fixed sink. By a configuration $c$ on $G$ we mean a placement of a number $c_{i}$ of "chips" or "grains of sand" on each nonsink vertex $i \in[n]$. A vertex $i$ is said to be unstable if the number of chips $c_{i}$ on $i$ is greater or equal to the degree of $i$. In this case, one can then "fire" the vertex $i$, distributing chips to each of its neighbors (one chip along every edge). Chips that are sent to the sink vertex disappear. It is easily seen that "firing" the vertex $i$ corresponds to subtracting the $i$-th column of the truncated Laplacian $\tilde{\mathcal{L}}(G)$ from $c$. If $G$ is connected, repeated chip-firing of vertices eventually leads to a stable configuration $\bar{c}$. One of the fundamental results for chip-firing games is that this configuration $\bar{c}$ is independent of the sequence of firings; we call $\bar{c}$ the stabilization of $c$.

A stable configuration $c$ on $G$ is said to be recurrent if $c$ has a nonnegative value on every nonsink vertex $[n]$, and if for any configuration $a$ there exists a configuration $b$ such that $a+b$ stabilizes to $c$. The stabilization process gives rise to a monoidal structure on the set of all configurations and forms a group when restricted to the recurrent configurations of $G$. This group is called the sandpile group of $G$, denoted $\mathcal{S}(G)$. The sandpile group can also be realized by considering the discrete subgroup $\mathbb{L}_{G} \subset \mathbb{Z}^{n}$ generated by the columns of the truncated Laplacian $\tilde{\mathcal{L}}(G)$. We then have an isomorphism

$$
\mathcal{S}(G) \cong \mathbb{Z}^{n} / \mathbb{L}_{G}
$$

given by $c \mapsto c+\mathbb{L}_{G}$. Thus, every element of $\mathbb{Z}^{n}$ is equivalent to a recurrent configuration modulo the reduced Laplacian. As a corollary to the Matrix-Tree theorem, we then see that the order of $\mathcal{S}(G)$ is given by the number of spanning trees of $G$.

The canonical configuration $c_{\omega}$ on $G$ is the maximally stable configuration given by

$$
\left(c_{\omega}\right)_{i}=|\{i j \in E: j \in[n]\}|-1
$$


for every nonsink $i \in[n]$. In the graph-theoretic Riemann-Roch theory developed in [1], configurations are naturally identified with "divisors" on the graph $G$. In this context the configuration $c_{\omega}-\mathbf{1}$ is closely related to the "canonical divisor" of $G$.

To fully describe our results we will need a few more notions from the chip-firing literature. As opposed to firing one vertex at a time, we consider a rule where one may fire sets of vertices simultaneously. This leads to a stronger version of stability and a resulting notion of superstable configurations. We will not detail the construction here as it will be enough for us to use the following characterization ([16]): a sequence $a=\left(a_{1}, a_{2}, \ldots, a_{n}\right)$ is a superstable configuration of $G$ if and only if $a$ is a $G$-parking function. For the next result, recall that an acyclic orientation of $G$ is an orientation $\mathcal{O}$ of the edges of $G$ with no directed cycles.

Theorem 1 ([4, Theorem 3.1]) There is a bijection between the set of acyclic orientations of $G$ with unique sink $n+1$ and the set of maximal superstable configurations of $G$. Given an acyclic orientation $\mathcal{O}$, the corresponding configuration $c=c^{\mathcal{O}}$ is given by

$$
c_{i}=|\{i \rightarrow \mathcal{O} j: j \in[n+1]\}|-1
$$

We note that the bijection described in [4] actually involves the set of acyclic orientations with unique source $n+1$, but of course there is a bijection between these and orientations with unique sink $n+1$ by reversing all arrows.

In [16] (Corollary 5.15) it is shown that a configuration $c$ is superstable if and only if $c_{\omega}-c$ is recurrent. Hence for an undirected graph $G$ on vertex set $[n+1]$, there is a bijective correspondence between minimal recurrent configurations, maximal superstable configurations, maximal $G$-parking functions, and acyclic orientations with $n+1$ as the unique sink vertex.

\section{Graphical arrangements and Whitney numbers}

In this section we introduce graphical hyperplane arrangements and other notions from geometric combinatorics that will serve to describe our resolutions. We describe the basic constructions and terminology here, and refer to [8] and [19] for more details.

Once again we fix our graph $G$ on vertex set $V=[n+1]$ with edge set $E$. For an edge $i j \in E$, we define a corresponding hyperplane

$$
h_{i j}:=\left\{x \in \mathbb{R}^{n+1}: x_{i}=x_{j}\right\}
$$

The arrangement $\mathcal{A}_{G}=\left\{h_{i j}: i j \in E\right\}$ of hyperplanes in $\mathbb{R}^{n+1}$ is called the graphical arrangement of $G$. The arrangement $\mathcal{A}_{G}$ dissects space into polyhedra, called cells, of various dimensions, and we use $f_{k}\left(\mathcal{A}_{G}\right)$ to denote the number of $k$-dimensional cells, or $k$-cells, for short. A flat of $\mathcal{A}_{G}$ is a nonempty intersection of elements of $\mathcal{A}_{G}$, and we let $L_{G}=L\left(\mathcal{A}_{G}\right)$ denote the collection of flats partially ordered by reverse inclusion. In fact $L_{G}$ is a ranked (geometric) lattice, called the lattice of flats, with minimum $\mathbb{R}^{n+1}$ and maximum $\hat{1}=\bigcap_{i j \in E} h_{i j}$. The $\operatorname{rank} \operatorname{rk}_{G}(x)$ of $x \in L_{G}$ is given 
by $n+1-\operatorname{dim} x$ and, since $G$ is connected, $L_{G}$ has total $\operatorname{rank} \operatorname{rk}(\hat{1})=n$ (which we also take to be the rank of $\mathcal{A}_{G}$ ). The lattice of partitions of $G$ is the collection of unordered partitions $V=V_{1} \uplus V_{2} \uplus \cdots \uplus V_{m}$ such that the vertex-induced graph $G\left[V_{k}\right]$ is connected for all $1 \leq k \leq m$. The partial order is by coarsening: a partition $\left\{V_{k}\right\}_{k}$ is smaller than $\left\{U_{l}\right\}_{l}$ if every $U_{s}$ is contained in some $V_{t}$. The lattice of partitions is naturally isomorphic to $L_{G}$ by associating to $\left\{V_{k}\right\}_{k}$, the flat

$$
\bigcap\left\{h_{i j}: i j \in E,\{i, j\} \subseteq V_{k} \text { for some } k\right\} \text {. }
$$

We will freely use both perspectives on the elements of $L_{G}$.

Central to the study of hyperplane arrangements (and more general matroids) is the notion of Whitney numbers. Here the doubly-indexed Whitney numbers of the first kind are given by

$$
w_{i j}\left(L_{G}\right)=\sum\left\{\mu_{L_{G}}(x, y): x, y \in L_{G}, \operatorname{rk}(x)=i, \operatorname{rk}(y)=j\right\}
$$

where $\mu_{L_{G}}$ is Möbius function of $L_{G}$. The Whitney numbers of $L_{G}$ are the simply indexed versions

$$
w_{j}\left(L_{G}\right)=w_{0 j}\left(L_{G}\right) .
$$

There is a well-known connection (see [8]) between the Whitney numbers of $L_{G}$ and the chromatic polynomial of $G$ given by

$$
\chi(t)=\sum_{j=0}^{n} w_{j}\left(L_{G}\right) t^{n-j}
$$

We say that a hyperplane $H$ is in general position with respect to the arrangement $\mathcal{A}_{G}$ if $\operatorname{dim}(x \cap H)=\operatorname{dim} x-1$ for all flats $x \in L_{G}$. If $\mathcal{A}$ is a hyperplane arrangement and $U$ an affine subspace not parallel to any hyperplane, then the restriction of $\mathcal{A}$ to $U$ is given by $\left.\mathcal{A}\right|_{U}=\{H \cap U: H \in \mathcal{A}\}$. We will need some further results from [8] that we collect here for future reference.

Theorem 2 ([8, Theorem 3.2]) Let $\mathcal{A}$ be an arrangement of linear hyperplanes of rank $r$ and let $k>0$. Let $H$ be a hyperplane general with respect to $\mathcal{A}$. Then the induced arrangement $\left.\mathcal{A}\right|_{H}$ has $|\mu(0,1)|=\left|w_{r}\left(L_{G}\right)\right|$ relatively bounded regions and $\left|w_{d-k, r}\right|$ relatively bounded $k-1$ cells.

Corollary 1 ([8, Corollary 7.3]) Let $G$ be a graph with vertex set $[n+1]$. The number of acyclic orientations of all contractions $G / S$ in which $S \in L_{G}$ has $k$ components, such that the vertex $n+1$ is the only sink, equals $\left|w_{n+1-k, n}\left(L_{G}\right)\right|$.

\section{Cellular resolutions from graphical arrangements}

In this section we describe our minimal cellular resolution of $M_{G}$ and derive some consequences. As above we fix a connected graph $G$ on vertex set $[n+1]$ and let 
Fig. 2 The complex $\mathcal{B}_{G}$ for our running example. The coordinates (as elements of $\mathbb{R}^{4}$ ) of the 0 -cells are indicated

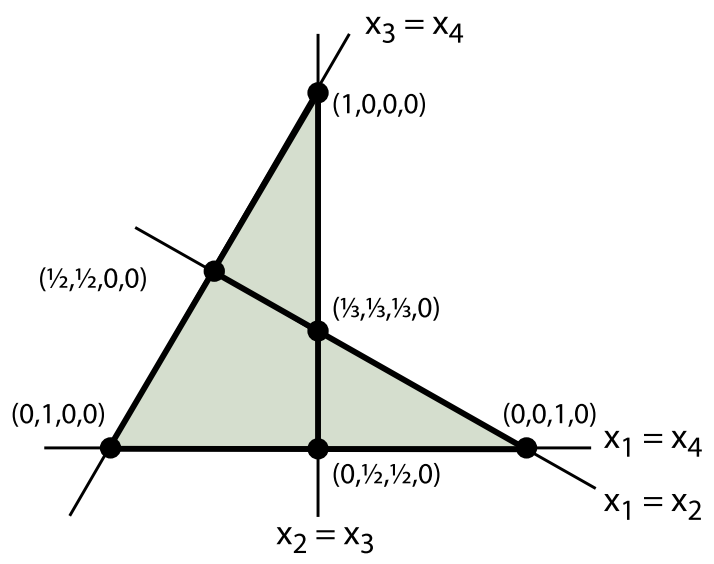

$\mathcal{A}_{G} \subset \mathbb{R}^{n+1}$ denote the associated graphical arrangement. Let $U \subset \mathbb{R}^{n+1}$ be the affine subspace

$$
U:=\left\{x \in \mathbb{R}^{n+1}: x_{n+1}=0, x_{1}+x_{2}+\cdots+x_{n}=1\right\} \cong \mathbb{R}^{n-1}
$$

We let $\tilde{\mathcal{A}}_{G}=\left\{\tilde{h}_{i j}:=h_{i j} \cap U: i j \in E\right\}$ be the restriction of $\mathcal{A}_{G}$ to $U$. Note that $\tilde{\mathcal{A}}_{G}$ is an essential arrangement of $|E|$ affine hyperplanes (two hyperplanes $\tilde{h}_{i j}$ and $\tilde{h}_{k l}$ coincide if and only if $i j$ and $k l$ are parallel).

For a point $p \in U$ such that $p_{i} \neq p_{j}$ for all $i j \in E$, we obtain an orientation on $G$ by orienting $i \rightarrow j$ if $p_{i}>p_{j}$. It is easy to see that this orientation is in fact acyclic. If $p$ takes the same value on some edges, we get an acyclic orientation on a certain contraction of $G$ as follows. From $p$ we get a partition of the vertex set $[n+1]=V_{1} \uplus V_{2} \uplus \cdots \uplus V_{s}$ where for each $k$ we place $i, j \in V_{k}$ if there is a path $i=i_{0} i_{1} \ldots i_{t}=j$ in $G$ such that $p_{i_{h-1}}=p_{i_{h}}$ for all $1 \leq h \leq t$. In particular, the induced subgraphs $G\left[V_{i}\right]$ are connected, and we denote by $G / p$ the result of contracting each $G\left[V_{i}\right]$ to a single vertex. The remaining edges satisfy $p_{i} \neq p_{j}$, and thus, we get an acyclic orientation on $G / p$.

The bounded complex $\mathcal{B}_{G}$ is the polyhedral complex of bounded cells of $\tilde{\mathcal{A}}_{G}$ in $U$. We let $\left|\mathcal{B}_{G}\right|$ denote the underlying pointset in $U$. The next result says that we can determine points $p$ of $\left|\mathcal{B}_{G}\right|$ in terms of the associated graph $G / p$ (Fig. 2).

Proposition 3 For $p \in U$ let $C$ be the inclusion-minimal cell having $p$ in the relative interior. Then $\operatorname{dim} C+2$ is the number of vertices of $G / p$, and $C \in \mathcal{B}_{G}$ if and only if the acyclic orientation on $G / p$ has a unique sink given by the vertex class containing $n+1$.

Proof Let $L \subset U$ be the intersection of all $\tilde{h}_{i j}$ for which $p_{i}=p_{j}$. Then $C$ is a full-dimensional cell in $L$, and we have to determine only $\operatorname{dim} L$. But the hyperplane arrangement induced in $L$ by $\tilde{\mathcal{A}}_{G}$ is isomorphic to the arrangement $\tilde{\mathcal{A}}_{G / p}$ and thus $\operatorname{dim} L=|V(G / p)|-2$. It thus suffices to assume that $p_{i} \neq p_{j}$ for all edges $i j \in E$. 


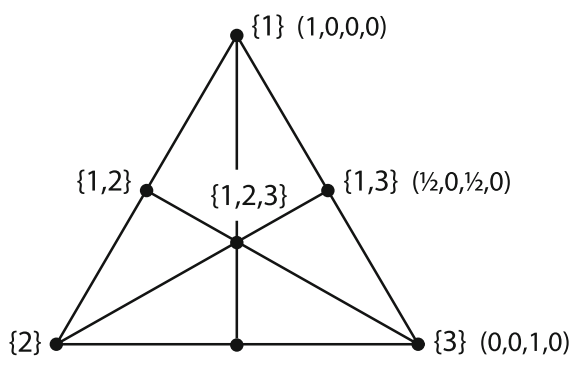

$\{2,3\} \quad(0,1 / 2,1 / 2,0)$

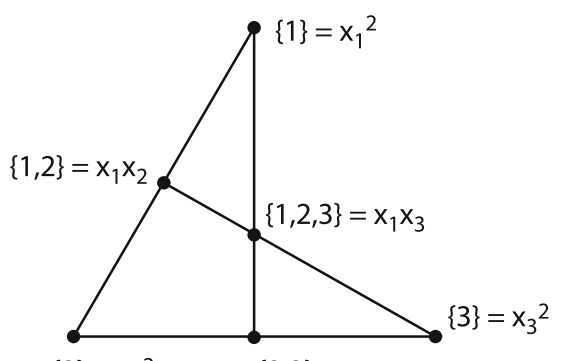

$\{2\}=x_{2}{ }^{2}$

$\{2,3\}=x_{2} x_{3}$

Fig. 3 The subset labeling of $\mathcal{B}_{K_{n+1}}$ corresponding to coordinates, and the induced monomial labeling of $\mathcal{B}_{G}$ for our running example $G$

If $G$ has more than one sink, let $i \neq n+1$ be one of them and let $j$ be an arbitrary source. We claim that $p$ is not contained in a bounded cell. Consider the halfline $\left\{p(t)=p+t\left(e_{j}-e_{i}\right): t \geq 0\right\}$. Since $j$ is a source, we have $p_{j}>p_{k}$ for all $j k \in E$ and $p(t)_{j} \geq p_{j}>p_{k} \geq p(t)_{k}$ for all $t \geq 0$. An analogous argument applies for $i$ and shows that the halfline is contained in the same inclusion-minimal cell of $\tilde{\mathcal{A}}_{G}$ as $p$.

Conversely, assume $C$ is unbounded. Since $C \subset U$, there is a point $q \in \operatorname{relint}(C)$ with $q_{i}<0$ for some $i$. All points in the relative interior of $C$ induce the same orientation on $G$. However, since $q_{n+1}=0>q_{i}$, and $G$ is connected, there is no directed path from $i$ to $n+1$, and thus, $n+1$ is not a sink.

In order for $\mathcal{B}_{G}$ to support a cellular resolution, we have to label the zerodimensional cells of $\mathcal{B}_{G}$. From Proposition 3, we infer that a 0 -cell $v$ of $\mathcal{B}_{G}$ is of the form $v=\frac{1}{|I|} e_{I}$ where $e_{I} \in\{0,1\}^{n+1}$ is the characteristic vector of the nonempty subset $I \subseteq[n]$. Using the fact that subsets $I \subseteq[n]$ correspond to monomials $m_{I}$ according to (2), this gives us a natural labeling of $\mathcal{B}_{G}$ (Fig. 3).

Corollary 2 Under the labeling described above, the 0 -cells of $\mathcal{B}_{G}$ are labeled by the minimal generators of $M_{G}$. The label $a_{v} \in \mathbb{N}^{n}$ of a 0 -cell $v=\frac{1}{|I|} e_{I}$ of $\mathcal{B}_{G}$ is given by

$$
\left(a_{v}\right)_{i}:=d_{I}(i)
$$

for all $i \in[n]$.

Proof From (2) and (3), we know that $\mathbf{x}^{a_{v}}=m_{I}$ is in $M_{G}$. Now, by Proposition $1, m_{I}$ is a minimal generator if and only if $G[I]$ and $G\left[I^{c}\right]$ are connected and, by Proposition 3 this is the case if and only if $v=\frac{1}{|I|} e_{I}$ is a 0 -cell of $\mathcal{B}_{G}$.

The labels on higher dimensional bounded cells are determined by the componentwise maximum of the labels of incident 0-cells. That is, for a cell $C \in \mathcal{B}_{G}$ and $i \in[n]$

$$
\left(a_{C}\right)_{i}=\max \left\{\left(a_{v}\right)_{i}: v \in C \text { is a } 0 \text {-cell }\right\} .
$$

However, we can also determine the labels of such cells directly. 
Proposition 4 Let $C \in \mathcal{B}_{G}$ be a bounded cell with label $a_{C} \in \mathbb{N}^{n}$. For $p \in \operatorname{relint}(C)$, we have

$$
\left(a_{C}\right)_{i}=\#\left\{i j \in E: p_{i}>p_{j}\right\}
$$

Proof Fix $1 \leq i \leq n$ and let $D_{i}(p):=\#\left\{i j \in E: p_{i}>p_{j}\right\}$. Consider the set $I \subseteq[n]$ of vertices of $G$ such that $k \in I$ if there is a path $k=i_{0} i_{1} \ldots i_{s}=i$ and $p_{i_{j}} \geq p_{i}$ for all $0 \leq j \leq s$. By construction $G[I]$ is connected. Assume that the complementary graph $G\left[I^{c}\right]$ is disconnected, and let $J \subset I^{c}$ be a connected component not containing $n+1$. Since $C$ is a cell in the hyperplane arrangement $\tilde{\mathcal{A}}_{G}$, and $p$ is a point in the relative interior, $C$ is the set of points $x \in U$ with

$$
x_{i}=x_{j} \text { if } p_{i}=p_{j} \quad \text { and } \quad x_{i} \geq x_{j} \text { if } p_{i}>p_{j}
$$

for all $i j \in E$. Observe that for all $t \geq 0$ the point $p(t)=p+\frac{t}{|I|} e_{I}-\frac{t}{|J|} e_{J}$ is in $C$. Indeed, $p(t) \in U$ for all $t$. As for the defining equations and inequalities of $C$, the only relevant case to check is $k l \in E$ with $k \in I$ and $l \in J$. By definition of $I$, we have $p_{k}>p_{l}$ and $p(t)_{k}=p_{k}+\frac{t}{|I|}>p_{l}-\frac{t}{|J|}$ for all $t \geq 0$. This shows that $C$ is not bounded.

It follows that $G\left[I^{c}\right]$ is connected and, by Proposition 3, the point $q=\frac{1}{|I|} e_{I}$ is a 0 -cell of $\mathcal{B}_{G}$. To see that $q \in C$ let $k l \in E$ such that $p_{k}>p_{l}$ but $q_{k}<q_{l}$. This implies $l \in I$ and $k \in I^{c}$, but since $p_{k}>p_{l}$, and there is a path from $l$ to $i$ with values $\geq p_{i}$, this means $k \in I$. As for monomial labels, we have $\left(a_{C}\right)_{i} \geq\left(a_{q}\right)_{i}=\#\{i j \in E: j \notin$ $I\}=D_{i}(p)$.

To see that $D(p)_{i}$ upper bounds $\left(a_{C}\right)_{i}$, observe that $\operatorname{relint}(C) \subseteq\left\{x \in U: x_{i}>\right.$ $x_{j}$ for all $i j \in E$ with $p_{i}>p_{j}$ \} for $i$ fixed. In particular $C$ is in the closure of this set and every point $q$ in the closure satisfies $D(q)_{i} \leq D(p)_{i}=\left(a_{C}\right)_{i}$.

Here is the main lemma that we need.

Lemma 1 Let $G=([n+1], E)$ be a connected graph and $\mathcal{B}_{G}$ the bounded subcomplex labeled according to the monomial ideal $M_{G}$. For every $\sigma \in \mathbb{N}^{n}$, the set

$$
\left|B_{G}\right|_{\leq \sigma}=\bigcup\left\{F \in B_{G}: a_{F} \leq \sigma\right\}
$$

is star-convex. Hence $\left|B_{G}\right|_{\leq \sigma}$ is contractible and in particular $\mathbb{K}$-acyclic for any field $\mathbb{K}$.

Proof Let $p^{1}, p^{2}, \ldots, p^{m}$ be the 0 -dimensional cells of $\left(\mathcal{B}_{G}\right)_{\leq \sigma}$ and for each $i$ let $a^{i}=a_{p^{i}} \in \mathbb{N}^{n}$ be the corresponding exponent vector of the monomial label. Let us define

$$
J=\bigcup_{i=1}^{m} \operatorname{supp}\left(a^{i}\right)
$$

In the subgraph of $G$ induced on the vertices $[n+1] \backslash J$, let $K$ be the set of vertices corresponding to the connected component containing the vertex $n+1$. Finally, define 
Fig. 4 The subspace $\left(\mathcal{B}_{G}\right)_{\leq \sigma}$ for our running example $G$ with $\sigma=(1,1,2)=x_{1} x_{2} x_{3}^{2}$. The star point in this case is $q=\left(\frac{1}{3}, \frac{1}{3}, \frac{1}{3}, 0\right)$

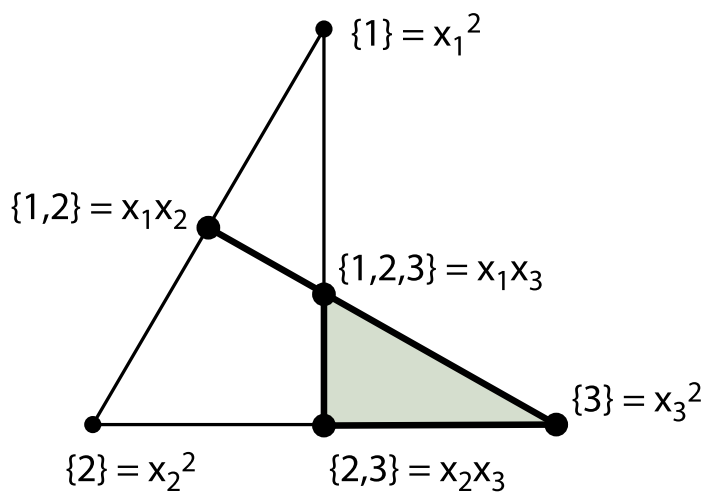

$I=[n+1] \backslash K \quad$ and $\quad q=\frac{1}{|I|} e_{I}$.

We claim that $q$ is a star point of $\left|\mathcal{B}_{G}\right|_{\leq \sigma}$. By construction, the contraction $G / q$ has a unique sink, and thus, $q$ is contained in the relative interior of some cell $\mathcal{B}_{G}$ with label $a_{q}$ (Fig. 4).

Let us next verify that $a_{q} \leq \sigma$ so that indeed $q \in\left|\mathcal{B}_{G}\right|_{\leq \sigma}$. First we claim that $I_{k}:=\operatorname{supp}\left(p^{k}\right) \subseteq I$ for all $k$ (note that $\operatorname{supp}\left(a^{k}\right) \subseteq \operatorname{supp}\left(p^{k}\right)$ ). For this suppose that $i \in I_{k}$. If $i \in \operatorname{supp}\left(a^{k}\right)$ then $i \in J$, and hence $i \in I$. If $i \notin \operatorname{supp}\left(a^{k}\right)$ then by definition $d_{I_{k}}(i)=0$. Since $p_{k}$ is a 0 -cell of $\mathcal{B}_{G}$, both $G\left[I_{k}\right]$ and $G\left[I_{k}^{c}\right]$ are connected. Therefore, any path from $n+1$ to $i$ has to include an edge $s t$ with $s \notin \operatorname{supp}\left(a^{k}\right)$ and $t \in \operatorname{supp}\left(a^{k}\right)$. Hence $i$ is not in the connected component of $n+1$ in the subgraph of $G$ induced on $[n+1] \backslash \operatorname{supp}\left(a^{k}\right)$. Since $\operatorname{supp}\left(a^{k}\right) \subseteq J$, this in turn implies that $i \notin K$ so that $i \in I$. Now let $i \in I$ with $\left(a_{q}\right)_{i}=d_{I}(i)>0$ and let $j \in I^{c}=K$ with $i j \in E$. Then there is a path from $n+1$ to $j$ that does not meet $J$. Hence, if $i \notin J$, there is a path in $J^{c}$ from $i$ to $n+1$ which would contradict $i \in I$. This implies $i \in J$ and thus $i \in I_{k}$ for some $k$. Now observe that $\sigma_{i} \geq d_{I_{k}}(i) \geq d_{I}(i)$.

Next, let $r \in\left|\mathcal{B}_{G}\right|_{\leq \sigma}$ be an arbitrary point. We need to show that the line segment connecting $r$ and $q$ is contained in $\left|\mathcal{B}_{G}\right|_{\leq \sigma}$. Recall that if $i \in I$, then either $d_{I}(i)=0$ or else, by Proposition 4, there exists a point $p \in\left|\mathcal{B}_{G}\right|_{\leq \sigma}$ such that $p_{i}>p_{j}$ for $i j \in E$. Thus, if $r_{i}>r_{j}$ for some $i j \in E$, we have $i \in I$ and $q_{i}=\frac{1}{|I|}$. But $q_{s} \leq \frac{1}{|I|}$ for all $s \in[n]$ and therefore $q_{i} \geq q_{j}$. We conclude that no hyperplane $\tilde{h}_{i j}$ strictly separates $r$ and $q$, and therefore, the open line segment $(r, q)$ is contained in some (inclusion minimal) cell $C$ of the arrangement $\tilde{\mathcal{A}}_{G}$.

We first confirm that $C \in \mathcal{B}_{G}$. Let $p \in(r, q) \subseteq \operatorname{relint}(C)$. Appealing to Proposition 3 , we need to show that the induced orientation on the contraction of $G / p$ has a unique sink given by the class containing the vertex $n+1$. By contradiction, assume that $i \in[n]$ corresponds to a sink in $G / p$ that is different from $n+1$. Let $i=i_{0} i_{1} \ldots i_{m}=n+1$ be a path such that $r_{i_{h-1}} \geq r_{i_{h}}$ for all $h=1, \ldots, m$. As $r$ is in the bounded subcomplex, and hence $G / r$ has a unique sink, such a path exists. By assumption, the path is not weakly decreasing for $p$; that is, there is an index $l$ with $p_{i_{l-1}}<p_{i_{l}}$. In particular we have $p_{i_{l}}>0$. We have $\operatorname{supp}(p)=\operatorname{supp}(r) \cup \operatorname{supp}(q)$ and, by construction, 
$\operatorname{supp}(r) \subseteq \operatorname{supp}(q)$ and hence $i_{l} \in I=\operatorname{supp}(q)$ and $q_{i_{l}}=\frac{1}{|I|}$. Thus, the path is weakly decreasing for $q$ which implies $p \in(r, q) \subseteq\left\{x \in U: x_{i_{l-1}} \geq x_{i_{l}}\right\}$. Recall that $U$ is the affine slice $U=\left\{x \in \mathbb{R}^{n+1}: x_{n+1}=0, x_{1}+x_{2}+\cdots+x_{n}=1\right\}$.

It is left to show that $a_{C} \leq \sigma$. For this let $i \in[n]$ with $p_{i}>0$, so that $i \in I$. If $r_{i}>0$ then since $\operatorname{supp}(r) \subseteq \operatorname{supp}(q)$, we have $q_{i}>q_{j} \Rightarrow r_{i}>r_{j}$ and thus $\sigma_{i} \geq\left(a_{r}\right)_{i} \geq\left(a_{C}\right)_{i}$. If $r_{i}=0$, then $p_{i}>p_{j} \Rightarrow q_{i}>q_{j}$ and thus $\sigma_{i} \geq\left(a_{q}\right)_{i} \geq\left(a_{C}\right)_{i}$, as desired.

Theorem 3 With the monomial labeling described above, the complex $\mathcal{B}_{G}$ supports a minimal cellular resolution of $M_{G} \subset \mathbb{K}\left[x_{1}, \ldots, x_{n}\right]$ over every field $\mathbb{K}$.

Proof We apply the criteria from Proposition 2. Corollary 2 and Lemma 1 imply that $\mathcal{B}_{G}$, with the monomial labeling described in Proposition 4, supports a cellular resolution of $M_{G}$. Proposition 4 asserts that the cellular resolution is indeed minimal.

Example 4 In the case of our running example, we obtain a free minimal resolution of $M_{G}$ from the labeled complex in Fig. 3. Disregarding the grading, the resolution has the form

$$
0 \leftarrow R \stackrel{\phi_{1}}{\longleftarrow} R^{6} \stackrel{\phi_{2}}{\longleftarrow} R^{8} \stackrel{\phi_{3}}{\longleftarrow} R^{3} \stackrel{\phi_{4}}{\longleftarrow} 0 .
$$

In particular the Betti numbers $\beta_{i}$ are given by the face numbers $f_{i-1}\left(\mathcal{B}_{G}\right)$, and the differentials are described by the incidence relations of $\mathcal{B}_{G}$.

In addition we obtain an explicit combinatorial formula for the Betti numbers of $I_{G}$, verifying a conjecture of Perkinson et al. [16, Conjecture 7.9].

Corollary 3 For a graph $G$ let $P_{k}$ denote the elements of $L_{G}$ that have $k$ components. For $S \in P_{k}$ let $G / S$ be the graph induced on $S$, with $k$ vertices given by contracting the elements of $S$, while preserving the edges between elements of $S$. Then the (nongraded) Betti numbers of the ideal $M_{G}$ are given by

$$
\beta_{k}\left(M_{G}\right)=\sum_{S \in P_{k+1}} \#\{c: \text { c a minimal recurrent configuration on } G / S\} .
$$

Proof Let $S$ be an element of $P_{k+1}$. As we have seen, the minimal recurrent configurations of $G / S$ correspond to the maximal $G / S$-parking functions, which are in turn in bijection with acyclic orientations of $G / S$ with unique sink $n+1$. From Proposition 3 we know that this set is in bijection with the $k-1$ cells of $\mathcal{B}_{G}$. Theorem 3 shows that $k-1$ cells of $\mathcal{B}_{G}$ index $\beta_{k}\left(M_{G}\right)$ (Fig. 5).

Corollary 4 If $G$ is a tree on the vertices $[n+1]$, then $M_{G}=\left\langle x_{1}, x_{2}, \ldots, x_{n}\right\rangle$, and $B_{G}$ is an $(n-1)$-dimensional simplex realizing the Koszul complex.

Proof In this case $\tilde{\mathcal{A}}_{G}$ is an arrangement of $n$ hyperplanes in $U \cong \mathbb{R}^{n-1}$. It is easy to see that the only acyclic orientation of $G$ with unique sink $n+1$ is obtained by orienting all edges toward $n+1$. And, since any contraction of $G$ is again a tree, we see that $\mathcal{B}_{G}$ has a unique bounded cell of dimension $n-1$ with $n$ facets, i.e., $\mathcal{B}_{G}$ is an $(n-1)$-dimensional simplex. 

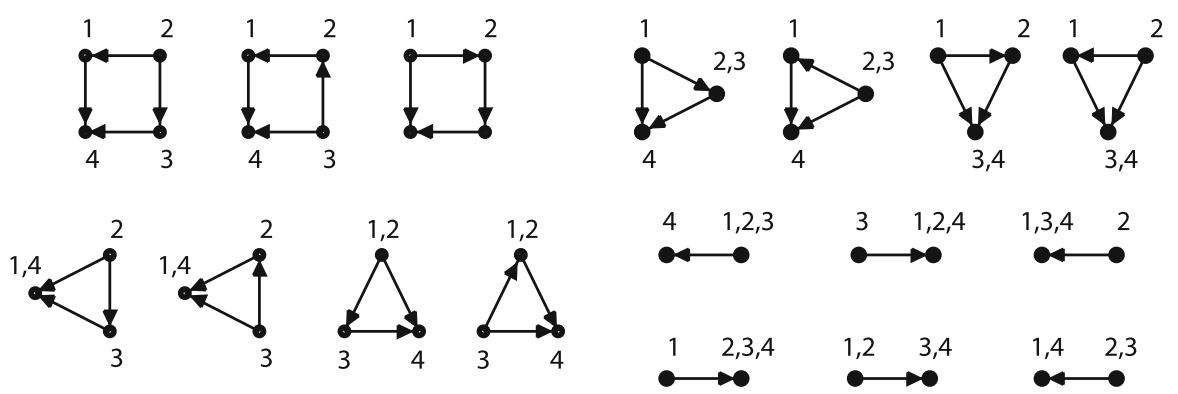

Fig. 5 The acyclic orientations of our example $G$ with vertex 4 as the unique sink, enumerating the syzygies of $M_{G}: \beta_{3}=3, \beta_{2}=8, \beta_{1}=6$

Lastly we note that, in principle, Theorem 3 gives an algebraic approach to acyclic orientations of $G$ and the study of the face poset of $\mathcal{B}_{G}$ by means of minimal $\mathbb{Z}^{n}$-graded resolutions. However, enumerating acyclic orientations of $G$ is \# $P$-hard [10], and we do not expect the algebraic method to be efficient.

\subsection{Duality}

As detailed in [12], the duality involved in the discrete Riemann-Roch theory of certain graphs $G$ has a commutative algebraic analog in terms of the ideal $M_{G}^{*}=M_{G}^{[\mathbf{k}+\mathbf{1}]}$. Here $M_{G}^{*}$ is the Alexander dual ideal of $M_{G}$ with respect to the monomial

$$
\mathbf{x}^{\mathbf{k}+\mathbf{1}}=x_{1}^{\operatorname{deg}(1)} x_{2}^{\operatorname{deg}(2)} \cdots x_{n}^{\operatorname{deg}(n)},
$$

where $\mathbf{1}=(1,1, \ldots, 1)$.

As a corollary to Theorem 3 we may apply the notion of duality of cellular resolutions [12] to obtain a (co)cellular minimal resolution of $M_{G}^{*}$. For this we will need the following result from the literature.

Proposition 5 ([13, Theorem 5.37]) Fix a monomial ideal I generated in degrees preceding a and a cellular resolution $\mathcal{F}_{X}$ of $R /\left(I+\mathbf{x}^{\mathbf{a}+\mathbf{1}}\right)$ such that all face labels on $X$ precede $\mathbf{a}+\mathbf{1}$. If $Y=\mathbf{a}+\mathbf{1}-X$, then $\mathcal{F}^{Y_{\preceq \mathbf{a}}}$ is a weakly cocellular resolution of $I^{[\mathbf{a}]}$. The resolution supported by $Y_{\preceq \mathbf{a}}$ is minimal if $\mathcal{F}_{X}$ is minimal.

In our context we take $G$ to be our graph on vertex set $[n+1]$, and let $\mathcal{B}_{G}$ denote the labeled polyhedral complex defined above. We define $\overline{\mathcal{B}}_{G}$ to be the colabeled polyhedral complex with underlying complex $\mathcal{B}_{G}$ but with monomial label on a face $C$ given by

$$
\left(\bar{a}_{C}\right)_{i}=\operatorname{deg}(i)+1-\left(a_{C}\right)_{i} .
$$

Combining our Theorem 3 with Proposition 5 gives us the following.

Proposition 6 Let $G$ be a graph on vertex set $[n+1]$ and set

$$
\mathbf{a}=(\operatorname{deg}(1), \operatorname{deg}(2), \ldots, \operatorname{deg}(n)) .
$$



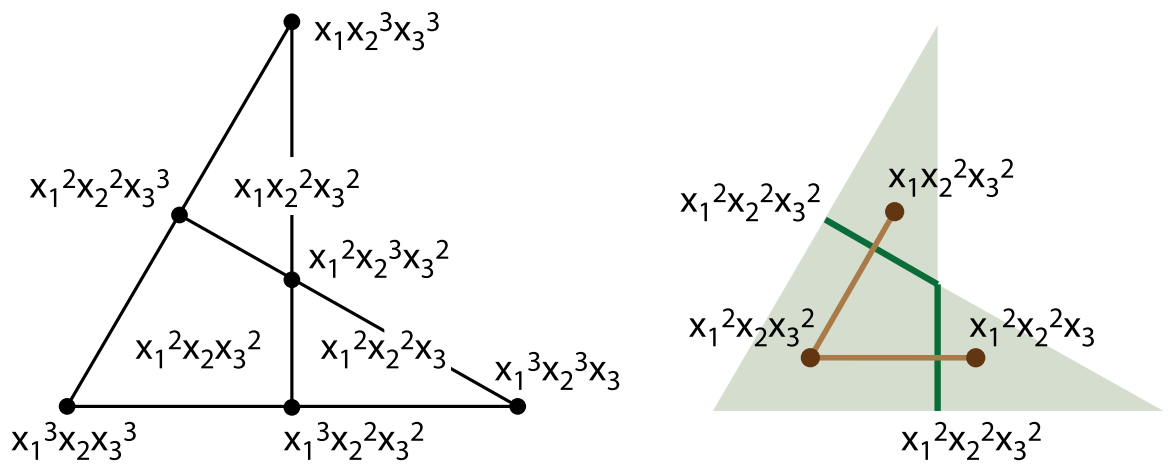

Fig. 6 The colabeled complex $\overline{\mathcal{B}}_{G}$ (with 0 -cells and 2-cells labeled) and the subcomplex $\left(\overline{\mathcal{B}}_{G}\right)_{\preceq \mathbf{a}}$, consisting of three 2-cells and two 1-cells. The dual complex is also depicted

Then with the notation established above, the labeled complex $\left(\overline{\mathcal{B}}_{G}\right)_{\preceq \mathbf{a}}$ supports a minimal cocellular resolution of the ideal $M_{G}^{*}$.

Example 5 In our running example, we have $\mathbf{a}=(2,2,2)$, and

$$
M_{G}^{*}=M_{G}^{\mathbf{a}}=\left\langle x_{1} x_{2}^{2} x_{3}^{2}, x_{1}^{2} x_{2} x_{3}^{2}, x_{1}^{2} x_{2}^{2} x_{3}\right\rangle
$$

The colabeled complex $\overline{\mathcal{B}}_{G}$ is depicted below, along with the subcomplex supporting the minimal resolution of $M_{G}^{*}$ (Fig. 6).

Remark 1 For the case of $G=K_{n+1}$, the complete graph on $n+1$ vertices, this is the duality between the (resolutions of the) tree ideals and the permutohedron ideals described in [13, Example 5.44].

\section{Further questions}

\subsection{Toppling ideals}

As mentioned in Sect. 2, there is a monomial term order $\preceq$ for $\mathbb{K}\left[x_{1}, \ldots, x_{n+1}\right]$ for which the ideal $M_{G}$ is the initial ideal of $I_{G}$. A natural question to ask is whether one can describe a minimal cellular resolution of the lattice binomial ideal $I_{G}$. A construction presents itself in terms of a quotient of the unimodular graphic lattice associated to $G$, similar to the cellular resolutions of binomial Lawrence ideals described in [2]. Mohammadi and Shokrieh [14] informed us about progress along these lines that will be published in a sequel to their recent paper.

\subsection{Monotone monomial ideals}

In [17] the authors study monotone monomial ideals, a class of monomial ideals that are strictly more general than those arising as $M_{G}$ for a graph $G$. We recall the definition 
here. A monotone monomial family $\mathcal{M}=\left\{m_{I}: I \in \Sigma\right\}$ is a collection of monomials indexed by a set $\Sigma$ of nonempty sets in $[n]$ that satisfy the conditions

(MM1) For $I \in \Sigma, \operatorname{supp}\left(m_{I}\right) \subseteq I$,

(MM2) For $I, J \in \Sigma$ such that $I \subset J$, we have $m_{I}$ divides $m_{J}$.

(MM3) For $I, J \in \Sigma, \operatorname{lcm}\left(m_{I}, m_{J}\right)$ is divisible by $m_{k}$ for some $K \supset I \cup J$ in $\Sigma$.

We then define the monotone monomial ideal $\langle\mathcal{M}\rangle$ associated to the family $\mathcal{M}$ to be the ideal generated by the monomials $m_{I}$ in $\mathcal{M}$.

The monomial ideals $M_{G}$ associated to a (directed) graph $G$ described above are monotone. In this case $\Sigma$ is the set of all nonempty subsets of $[n]$, and $m_{I}$ is given by the formula 2 . The natural question to ask is if a similar construction to $\mathcal{B}_{G}$ can be used to resolve the ideals $\langle\mathcal{M}\rangle$. Is there an arrangement of hyperplanes corresponding to a monotone family?

\subsection{Shi arrangements and duality}

In his study of the affine Weyl group of type $A_{n-1}$, Shi introduced the arrangement $\mathcal{S}_{n}$ of hyperplanes in $\mathbb{R}^{n}$ that now bears his name:

$$
\mathcal{S}_{n}=\left\{x_{i}-x_{j}=0,1: 1 \leq i<j \leq n\right\} .
$$

Shi proved that the number of regions in the complement of $\mathcal{S}_{n}$ is given by $(n+$ $1)^{n-1}$ (the number of trees on $n+1$ labeled vertices). Stanley [18] gave the first bijective proof of this fact by providing an explicit labeling of the regions with parking functions. Hopkins and Perkinson [9] generalize this picture, motivated by a conjecture of Duval, Klivans, and Martin. Associated to a graph $G$ they define what they call a bigraphical arrangement and show that a Pak-Stanley type labeling of its regions is in bijection with the $G$-parking functions. Specifying certain parameters of the bigraphical arrangements recover the $G$-Shi and $G$-semiorder arrangements.

From Theorem 3, we obtain a minimal resolution of the ideal $M_{G}$ from the graphical arrangement of $G$. We also know that when $M_{G}$ is Riemann-Roch (in the sense of [12]), the generators of $M_{G}^{*}$ (the Alexander dual of $M_{G}$ ) are given by the maximal $G$ parking functions. It would be interesting to find a connection between the bigraphical arrangements of [9] and the cellular resolutions that we have considered here.

\subsection{Topology of the partition poset}

Björner and Wachs [5] use the graphical hyperplane arrangement of the complete graph (the so-called braid arrangement) to give an explicit basis for the homology of the partition poset. It would be interesting to connect this study to the resolutions of the ideals studied here, and in particular to consider the case of a general graph $G$.

Acknowledgments We would like to thank Michelle Wachs, Christian Haase, Bernd Sturmfels, Katharina Jochemko, and Francesco Grande for stimulating discussions. We thank the two anonymous referees for very helpful comments and corrections. 


\section{References}

1. Baker, M., Norine, S.: Riemann-Roch and Abel-Jacobi theory on a finite graph. Adv. Math. 215, 766-788 (2007)

2. Bayer, D., Popescu, S., Sturmfels, B.: Syzygies of unimodular Lawrence ideals. J. Reine Angew. Math. 534, 169-186 (2001)

3. Bayer, D., Sturmfels, B.: Cellular resolutions of monomial modules. J. Reine Angew. Math. 502, 123-140 (1998)

4. Benson, B., Chakrabarty, D., Tetali, P.: $G$-parking functions, acyclic orientations and spanning trees. Discret. Math. 310, 1340-1353 (2010)

5. Björner, A., Wachs, M. L.: Geometrically constructed bases for homology of partition lattices of type $A, B$ and $D$, Electron. J. Combin., 11, pp. Research Paper 3, 26 (2004/06)

6. Gabrielov, A.: Abelian avalanches and Tutte polynomials. Physica A 195, 253-274 (1993)

7. Godsil, C., Royle, G.: Algebraic graph theory. Graduate Texts in Mathematics, vol. 207. Springer, New York (2001)

8. Greene, C., Zaslavsky, T.: On the interpretation of Whitney numbers through arrangements of hyperplanes, zonotopes, non-radon partitions, and orientations of graphs. Trans. Am. Math. Soc. 280, 97-126 (1983)

9. Hopkins, S., Perkinson, D.: Bigraphical arrangements. Trans. Am. Math. Soc. (to appear)

10. Linial, N.: Hard enumeration problems in geometry and combinatorics. SIAM J. Algebraic Discret. Methods 7, 331-335 (1986)

11. Manjunath, M., Schreyer, F.-O., Wilmes, J.: Minimal free resolutions of the $G$-parking function ideal and the toppling ideal. Trans. Am. Math. Soc. (to appear). arXiv:1210.7569

12. Manjunath, M., Sturmfels, B.: Monomials, binomials, and Riemann-Roch. J. Algebr. Comb. 37, 737756 (2013)

13. Miller, E., Sturmfels, B.: Combinatorial commutative algebra. Graduate Texts in Mathematics, vol. 227. Springer, New York (2005)

14. Mohammadi, F., Shokrieh, F.: Divisors on graphs, connected flags, and syzygies. Internat. Math. Res. Notices (to appear). arXiv:1210.6622

15. Peeva, I., Sturmfels, B.: Generic lattice ideals. J. Am. Math. Soc. 11, 363-373 (1998)

16. Perkinson, D., Perlman, J., Wilmes, J.: Primer for the algebraic geometry of sandpiles. In: Tropical and Non-Archimedean Geometry, Contemporary Mathematics, vol. 605. American Mathematical Society, Providence, RI (2013). arXiv:1112.6163

17. Postnikov, A., Shapiro, B.: Trees, parking functions, syzygies, and deformations of monomial ideals. Trans. Am. Math. Soc. 356, 3109-3142 (2004). (electronic)

18. Stanley, R.P.: Hyperplane arrangements, parking functions and tree inversions, in Mathematical essays in honor of Gian-Carlo Rota (Cambridge, MA, 1996), vol. 161 of Progr. Math.,Birkhäuser Boston, Boston, MA, pp. 359-375 (1998)

19. Stanley, R.P.: An introduction to hyperplane arrangements. In: Geometric Combinatorics, IAS/Park City Mathematics Series, vol. 13. American Mathematical Society, Providence, RI, pp. 389-496 (2007) 